\title{
Design of Phone Anti-obsessed System Based on the User Behavior
}

\author{
Xiafu Pan \\ Information Technology Department \\ Hainan Vocational College of Political Science and Law \\ Haikou, China \\ pxf_2008@126.com
}

\begin{abstract}
Traditional anti-obsessed system is only use for identity management, but it cannot be used for entertainment conduct analysis, and cannot be able to accurately identify entertainment software. This paper design a new mobile phone Anti-obsessed system based on the user behavior. The system can dynamic capture user's interactive behavior data, and uses these data for detecting whether the software is in the entertainment software classification. From the result of comparing with interaction behavior threshold number of classification, mobile phone Anti-obsessed system can decide whether to block the use of entertainment software. Experiment showed that phone Anti-obsessed system could effectively limit the time playing mobile phone by young people and provide a new approach to prevent adolescent's indulged mobile phone application.
\end{abstract}

Keywords: Android; Anti-obsessed system; App; user behavior; application classification;

\section{INTRODUCTION}

With the prevalence of mobile devices, mobile phone applications develop rapidly. Many users, in particular, minors indulge in the use of mobile phones, result in physical and psychological harm. Therefore, the use of mobile phone software for minors must be controlled. There are two ways to control: manual monitoring and software monitoring. Software monitoring system uses software to monitor the user's behavior, and limits the user's application. It's referred to as the Anti-obsessed system [1]. However, whether the IOS system's App Store, or the Google Play market, for minors to download and play the game no special settings and controls. So the mobile phone Anti-obsessed system plays its effective active role for limiting adolescents indulged.

\section{EXISTING ANTI-OBSESSED SYSTEM}

The mobile phone Anti-obsessed system mainly originates from the network game Anti-obsessed system. The network game business units adopt technical measures to reject minors who are not suitable for this game or to limit the game time when a user's online time is too long [1]. Network game Anti-obsessed system is basically consists of 3 parts: registration subsystem, authentication subsystem and background limit subsystem. A player must enter personal identification number to registration system before landing game, so authentication system can verify user's information, and background limit system restricts user's activities. Through the system, User that identity is minors, information is not standardized and is not pass the

This paper supported by the Hainan Provincial Natural Science Foundation of China (No. 20156244). validation, cannot successfully landing the game; player on line reaches the limit of time cannot get game awards, and so forth [2].

But there are many shortcomings in the existing network Anti-obsessed system. Firstly, mobile phone games software have many types, there are very few mobile phone user playing with only one game, and even player in same game has more than one account, so the cross-use increases time of using mobile phone. Secondly, most network Anti-obsessed system is only for online games behavior, not for offline software behavior, many console game cannot be monitored. Thirdly, the existing anti-obsessed system is only used on computer, and not on mobile phone. Phone system each time allows only one program run in the foreground, and uses record status mode to switch programs [3]. So mobile phone software and computer software operating mechanism is different. To distinguish between them, mobile phone is also known as application, hereinafter referred to as App. Therefore, in order to avoid these problems, Anti-obsessed system of mobile phone must be able to detect App features, and can classify entertainment applications to together manage by using the same restriction.

There are two main ways of software features detection: one is software behavior detection, the other is user and software interaction behavior detection. Current research on software behavior detection focuses on malware detection. The main method of malware detection is to establish some kind of test model, and then determines software behavior abnormal according to the model calculation. For instance, a propagation model based on reverse web graph [4], a Markov chain model [5], a statistical data compression model of Dynamic Markov Compression [6]. Another way of software behavior detection can also adopt identification algorithm that based on iterative, classification and threshold comparison [7]. But these models or algorithms are more complex, require a lot of computing time, not suitable for mobile phone system. Application detection of phone system is divided into two types, the use of static analysis method based on predefined abnormal or normal behavior template, and the dynamic methods based on phone application features. For Android system, Padriya and Polla [8, 9] adopt static analysis techniques of Android source code to detection, the others authors also adopt more special features for analysis, Dalvik command system [10], permissions model frequent excavation [11], permission sequential pattern mining algorithm [12], Android system call [13], and so forth. Shabtai [14] extracts the dynamic characteristics by artificial methods, and uses characteristics classification 
for dynamic detection. And some authors adopt the static and dynamic combine algorithm, such as Triple Hybrid Ensemble Algorithm (THEA) which is considered multiclass Android features [15]. These application behavior detection methods are accurate and effective. However entertainment software is harmless for the system, malware detection cannot distinguish functions of software. This entertainment software require user to operate frequently, so user behavior can be used as indicators for monitoring. Through the user behavior classification, software can be classified different types. By classification, Anti-obsessed system can limit using time of software, and thus achieves effect of anti-indulgence.

\section{ANTI-OBSESSED SYSTEM OF ANDROID PHONE BASED ON USER BEHAVIOR}

Based on the above discussion of Anti-obsessed system, this paper presents a new design of mobile phone Antiobsessed system. This system is based on user interaction behavior, it can identify and classify interaction behavior accurately, and can limit the use time of application by using application category.

\section{A. System implementation environment}

In recent years, Android platform has become a very popular mobile phone operating system platform, and takes up most of the mobile operating system market. As an open mobile operating system, Android has a certain degree of representativeness [3]. So the mobile phone Anti-obsessed system chooses Android system for design environment. This system adopts Windows 7 for operating system, open-source software Eclipse Juno $4.2+$ Jdk $8+$ Android SDK 4.4 for programming environment, and Google Nexus 5 series mobile phone for hardware.

\section{B. Overall system design}

The mobile phone Anti-obsessed system structure is shown in Fig .1. System chooses Android background service process for timing collecting interaction data, such as information of user's press keys, gestures and sensors. By this information, application can be classified in accordance with specific rules. If application is in entertainment classification, system adopts shutdown process to limit use time of this application.

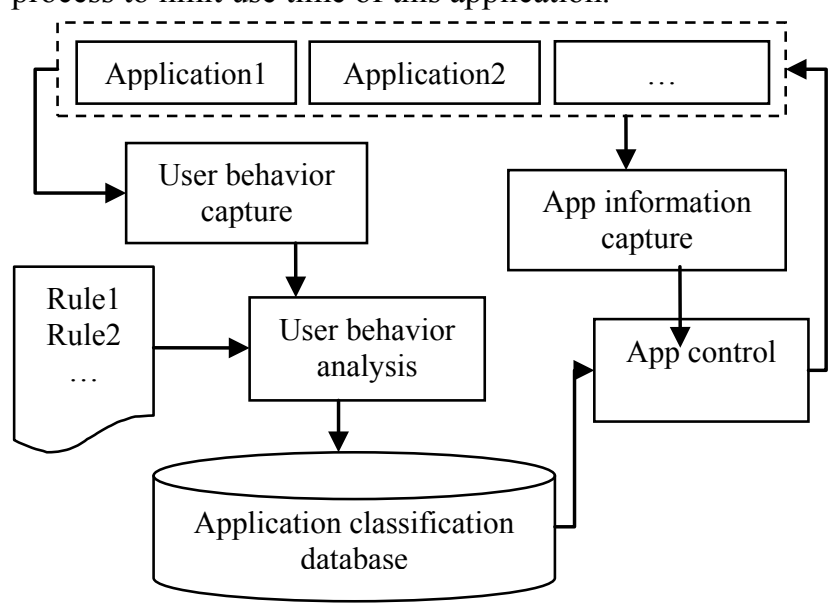

Figure 1. Anti-obsessed system model diagram.
System is divided into 4 modules: user interaction behavior capture module, user behavior analysis module, App information capture module, App control module. These modules complete the functions of mobile phone Anti-obsessed system.

User interaction behavior capture module collects user behavior information of current activity application, sorts user behavior data, and delivers data to the user behavior analysis module. This module runs in the background, and chooses the android.content.BroadcastReceiver component for real-time listening.

User behavior analysis module uses large amount application and user interaction data as test sample for setting the library rules of application classification. Then the module classifies applications by these rules, and stores this application information in the application classification database. This module is the core of whole system.

App information capture module is mainly responsible for application information acquisition, including application name, use of time and whether network is used, and passes information to the App control module. Module chooses the com.android.internal.os.PkgUsageStats class to obtain applications information.

App control module determines whether or not to allow the mobile phone application, according to the phone App name and classification information of the application classification database. If this is not allowed, the module uses the android.app.ActivityManager class to close the application.

\section{Application and user interaction rules}

\section{1) The user interaction behavior data}

The user interaction rules are based on the mode of user's actions. In general, application's operations have a certain degree of regularity. Especially, a lot of game applications use repetitive mechanical operation for games playing. Application classification rules are the results of these regular patterns. Through large amounts of data training sample, delineation of the threshold, Antiobsessed system can provide accurate application classification rules.

User's interactions with mobile phone are mainly three of the following: key operation, touch screen gesture operation and sensor operation. In order to provide accurate phone classification rules, interaction operations are set to more detailed classification. So, system can determine features of application accurately by classification rules.

Key operation of mobile phone include: function keys operation and character keys operation. Acquisition Keys press operation is only capture the input number within a certain period of time, does not capture specific keys.

Gesture operation is more complex. In particular some applications have their own specific gestures. Therefore application classification can only choose touch points of gesture operation. Touch points of screen gestures are classified with single-touch and multi-touch. Gesture acquisition major is the number of these two gesture operations.

Many sensors are used on mobile phone, such as orientation sensor, magnetic field sensor, temperature sensors, light sensor and pressure sensor. However, not 
every mobile phone have all of these sensors, so sensor operation acquisition sets them as one classification, and only records the total number of operations of each sensor.

Therefore, user interaction behavior is divided into 5 classes, the number of interaction operations can be computed by following formula:

$$
\varepsilon_{\alpha}=\frac{\sum_{i=1}^{n} d_{\alpha, i}}{n} \quad(\alpha=1,2,3,4,5)
$$

$d_{\alpha, i}$ is interaction operation count within 5 minutes, $n$ is number of data acquisition, and generally $n=10, \alpha$ represents the type of interaction, ranges from 1 to 5 , corresponds to each classification of user interaction behavior.

Statistical results of mobile phone application's user interaction are shown in TABLE I.

TABLE I. THE NUMBER OF OPERATIONS

\begin{tabular}{cccccc}
\hline & \multicolumn{2}{c}{ keys } & \multicolumn{2}{c}{ gestures } & sensors \\
& $\begin{array}{c}\text { function } \\
\text { keys }\end{array}$ & $\begin{array}{c}\text { character } \\
\text { keys }\end{array}$ & $\begin{array}{c}\text { single- } \\
\text { touch }\end{array}$ & $\begin{array}{c}\text { multi- } \\
\text { touch }\end{array}$ & \\
\hline Network game & 3.5 & 0 & 25.6 & 27.7 & 0 \\
Stand-alone game & 2.2 & 0 & 38.8 & 28.5 & 20.6 \\
Video application & 12.0 & 0 & 7.7 & 0 & 0 \\
Audio application & 11.2 & 0 & 5.6 & 0 & 0 \\
Browser & 14.6 & 39.7 & 5.0 & 5.2 & 1.4 \\
Chat application & 1.0 & 73.6 & 7.8 & 0 & 2.4 \\
\hline
\end{tabular}

In TABLE I, mobile phone application classification is based on the Google Play market's application categories [7].

We choose specific template with the following:

Network game: Hearthstone, Gunz dash for Wechat, Thunder aircraft.

Stand-alone game: Kingdom Rush, Plants VS Zombies, Plague Inc.

Video application playback: WMP, Youku, QQ player, Tudou video.

Audio application: QQ music, Baidu music.

Browser: Chrome, Firefox.

Chat application: QQ, WeChat.

\section{2) The user interaction behavior rules}

According to statistics, different mobile phone applications have different number of interaction operation. In general, games application requires more of gestures or sensor operation, browser and chat application require more character keys to enter, the audio and video application operation is very small, and most only need to press the function key. So it can be set a threshold, by this value, the application possible to determine whether it belongs to a rule. The threshold is defined as $t_{\alpha}$, its value is based on experience, see TABLE II. And the formula that uses to determine application classification rules can be got by following:

$$
T_{\alpha}=\varepsilon_{\alpha}-\sigma_{\alpha}-t_{\alpha}
$$

$\varepsilon_{\alpha}$ is defined by (1). $\sigma_{\alpha}$ is variance of interaction operations number, mainly uses to prevent the bias in statistical process too large, and defines as follow:

$$
\sigma_{\alpha}=\frac{\sqrt{\sum_{i=1}^{n}\left(d_{\alpha, i}-\varepsilon_{\alpha}\right)^{2}}}{n}
$$

$\varepsilon_{\alpha}, d_{\alpha, i}, \alpha, n$ of (2) also has the same definition of (1).

TABLE II. MOBILE PHONE APPLICATION OPERATION THRESHOLD

\begin{tabular}{ccccc}
\hline \multirow{2}{*}{ operations } & threshold & \multicolumn{2}{c}{ rules } \\
& & $t_{\alpha}$ & $T_{\alpha} \geq 0$ & $T_{\alpha}<0$ \\
\hline \multirow{2}{*}{ keys } & function keys & 10 & $K 1 \_H$ & $K 1 \_L$ \\
& character keys & 30 & $K 2 \_H$ & $K 2 \_L$ \\
gestures & single-touch & 20 & $S 1 \_H$ & $S 1 \_L$ \\
\multirow{2}{*}{ sensors } & multi-touch & 30 & $S 2 \_H$ & $S 2 \_L$ \\
& & 10 & $C \_H$ & $C \_L$ \\
\hline
\end{tabular}

The application classification rules are logical combination of the rules in TABLE II. If the application meets logic, system can determine it into one class. Application classification rules are shown in TABLE III. There two common classifications of application: work application and entertainment application. Entertainment applications are classified a total of four: game, audio and video, browser, chat application. If application does not belong to entertainment application, then it can be divided

\begin{tabular}{|c|c|c|}
\hline application c & ssifications & rules \\
\hline $\begin{array}{c}\text { Entertainment } \\
\text { application } \\
\text { work application }\end{array}$ & $\begin{array}{c}\text { game } \\
\text { audio \& video } \\
\text { browser } \\
\text { chat app }\end{array}$ & 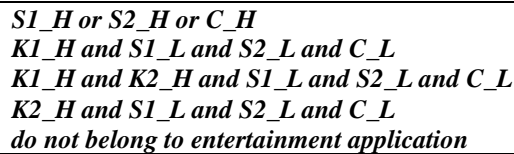 \\
\hline
\end{tabular}
into the work application.

TABLE III. APPLICATION CLASSIFICATIONS RULES

3) User behavior analysis algorithm

The user behavior analysis algorithm is described as follow:

Step 1: for each interaction classifications (For $\alpha=1$ to 5).

a. receive $d_{\alpha, i}$ from user interaction behavior capture module.
b. compute $\varepsilon_{\alpha}$.
c. compute $\sigma_{\alpha}$.
d. compute $T_{\alpha}$.

Step 2: use $T_{\alpha}(\alpha=1 \cdots 5)$ to determine whether compliance with the rules in TABLE II.

Step 3: use TABLE III to get application classification.

Step 4: save classification information to application classification database.

\section{Application classification database}

Application classification database is provided by SQLite Database. The SQLite Database is a lightweight database, which is specifically used for resource-limited device (such as mobile phone, PDA), and it is a data file of in bottom of Android system. Application gets a specified database object, uses this object to operate database [16].

Application classification database mainly storages application name, classification, using time, and control scheme. By different control schemes, the monitoring activities of application can be implemented in different 
way. For work application, system will give a reminder if the user used for a long period of time. For entertainment software, if used for a long period of time, the application will be forced to close.

\section{EXPERIMENT RESULT AND ANALYSIS}

Application test samples were randomly downloaded from Google Play, and use the Google Play application categories to classify. The selection of applications is to cover all types of entertainment classification, and focus on game applications the selection of applications is to cover all types of entertainment classification, and focus on game applications (total 563, accounting for $73.7 \%$ of the entertainment applications). User behavior analysis algorithm calculates results as following:

TABLE IV. APPLICATION CLASSIFICATION DETECTION RESULTS

\begin{tabular}{cccc}
\hline application classification & quantity & detection & success rate \\
\hline entertainment application & 790 & 764 & $96.7 \%$ \\
work application & 532 & 520 & $97.7 \%$ \\
total & 132 & 1284 & $97.1 \%$ \\
\hline
\end{tabular}

From the experiment, more than $97 \%$ of application can be properly to detect. And for some unable to identify applications, the Anti-obsessed system can add them to application classification database by using black-andwhite list.

\section{CONCLUSION}

The mobile phone Anti-obsessed system focuses on user's interaction behavior analysis, and more accurately identify entertainment application by using a threshold value comparison algorithm. The application classification algorithm that is used to anti-obsessed is making up for the deficiencies in existing phone anti- obsessed system. Experiment result indicates that phone Anti-obsessed system prevents a new approach for adolescent's indulged application.

\section{REFERENCES}

[1] G. Di, C. Yan, S. Zhang, et al, Analysis on the Internet Addiction and Anti-obsession Software, Applied Mechanics and Materials, no. 39, pp. 38-43, 2011.
[2] L. Wang, The Study of The Anti-Obsession System Based on Distributed Database Technology, Electronic Test, no. 14, pp. 41, 48-49, 2014.

[3] B. Hardy, B. Phillips, \{ \it Android Programming: the Big Nerd Ranch Guide\}, Addison--Wesley Professional, 2014

[4] C. Liu, Z. Wang, C. Jia, A Model of Malware Passive Propagation on the WWW, Journal of Computational Information Systems, vol. 7, no. 7, pp. 2593-2600, 2011.

[5] N. YE, A Markov chain model of temporal behavior for anomaly detection: Proc of IEEE Systems, Man, and Cybernetics Information Assurance and Security Work-shop, New York: IEEE Computer Society, pp. 171-174, 2000.

[6] W. Deng, Q. Liu, H. Cheng, etal, A Malware Detection Framework Based on Kolmogorov Complexity, Journal of Computational Information Systems, vol. 7, no. 8, pp. 2687-2694, 2011.

[7] Y. Yan , J. Chen , J. Cai, Model for Preventing Indulgence Base on Process Behavior. Journal of Nanchang University (Natural Science), vol. 37, no. 2, pp. 290-293, 2013.

[8] Mariantonietta L. Polla, F. Martinelli, D. Sgandurra, A Survery on Security for Mobile Devices, IEEE communication Surveys $\backslash \&$ Tutorials, vol. 15, no. 1, pp. 446-471, 2013.

[9] P. Nitin, M. Nilay. Review of behavior malware analysis for Android. International Journal of Enagineering and Innovative Technology, vol. 2, no. 7, pp. 230-232, 2013.

[10] T. Li, H. Dong, C. Yuan, et al, Description of Android Malware Feature Based on Dalvik Instructions, Journal of Computer Research and Development, vol. 51, no. 7, pp. 1458-1466, 2014.

[11] H. Yang, Y. Zhang, Y. Hu, et al, Android Malware Detection Method Based on Permission Sequential Pattern Mining Algorithm, Journal on Communications, vol. 34, no. Z1, pp. 106-115, 2013.

[12] L. Lei, J. Jing, Y. Wang, et al, A Behavior-Based System Resources Access Control Scheme for Android, Journal of Computer Research and Development, vol. 51, no. 51, pp. 10281038, 2014.

[13] Z. Cai, X. Peng, Detection of Android malware based on system calls, Computer Engineering and Design, vol. 34, no. 11, pp. $3757-$ 3761, 2013

[14] S. Asaf, K. Uri, E. Yuval, et al. Andromaly: A behavioral malware detection framework for Android devices. Journal of Intelligent Information Systems, vol. 38, no. 1, pp.161-190, 2012.

[15] H. Yang, Y. Zhang, Y. Hu, et al, A Malware Behavior Detection System of Android Applications Based on Multi-Class Features, Chinese Journal of computer, vol. 37, no. 1, pp. 15-25, 2014.

[16] Google Developers Live, Package Index - Android SDK, Android Community Docs, http://www.androidcommunitydocs.com/reference/packages.html, 2013.J. Clerk Maxwell, A Treatise on Electricity and Magnetism, 3rd ed., vol. 2. Oxford: Clarendon, 1892, pp.68-73. 